
Supporting Information For Manuscript ie050055f:
8 Pages

\title{
Two new parameters for predicting the entropy of melting: Eccentricity $(\varepsilon)$ and Spirality $(\mu)$
}

\author{
Jennifer L.H. Johnson* and Samuel H. Yalkowsky \\ The University of Arizona College of Pharmacy \\ Department of Pharmaceutical Sciences \\ 1703 E. Mabel St. Tucson, Az. 85721 \\ jjohnson@pharmacy.arizona.edu
}

*phone: 520-626-2014

*Fax: 520-626-4063

*e-mail: jjohnson@pharmacy.arizona.edu or jjohnson@imarx.com 


\section{SUPPORTING INFORMATION}

\section{$\underline{\text { Appendix }}$}

\section{Compound Name}

1,1-diphenyldodecane

1,2,3-trimethylbenzene

1,2,4-trimethylbenzene

1,3,5-trimethylbenzene

1,2,3,4-tetramethylbenzene

1,2,3,5-tetramethylbenzene

1,2,4,5-tetramethylbenzene

Anthanthrene

Anthracene

Benz[a]anthracene

Benzene

Benzo[a]naphthacene

Benzo[a]pentacene

$\mathbf{M P}_{\text {exp }}$
(Kelvin)

281

248

229

228

265

249

352

537

489

433

279

536

630
$\Delta \mathbf{H}_{\text {exper }}$

(kJxK/mol)

41

8

3.76

9.5

11.23

12.9

21

13.19

0.03744

25.55

0.047579

29

18.07

0.036949

21

24.03

0.055497

10

6.15

0.022083

29.99

0.055951

35.95

0.057063 


\begin{tabular}{|c|c|c|c|c|}
\hline Benzo[a]perylene & 388 & & 30.75 & 0.079253 \\
\hline Benzo[a]pyrene & 450 & 26 & 24.79 & 0.055107 \\
\hline Benzo[b]chrysene & 566 & & 29.99 & 0.052986 \\
\hline Benzo[b]perylene & 474 & & 30.75 & 0.064942 \\
\hline Benzo[c]chrysene & 399 & 16 & 29.99 & 0.075163 \\
\hline Benzo[c]phenanthrene & 341 & & 24.03 & 0.070469 \\
\hline Benzo[c]picene & 732 & & 35.95 & 0.049112 \\
\hline Benzo[e]pyrene & 451 & 16 & 24.79 & 0.054924 \\
\hline Benzo[g]chrysene & 387 & & 29.99 & 0.077494 \\
\hline Benzo[ghi]perylene & 551 & & 25.55 & 0.046345 \\
\hline Benzo[h]pentaphene & 519 & & 35.95 & 0.069268 \\
\hline Chrysene & 529 & 26.15 & 24.03 & 0.045443 \\
\hline Corannulene (bowl-shaped) & 542 & 17 & 20.35 & 0.037546 \\
\hline Coronene & 711 & 19.2 & 26.31 & 0.037004 \\
\hline Dibenz[a,c]anthracene & 479 & 26 & 29.99 & 0.062649 \\
\hline Dibenz[a,h]anthracene & 539 & 31 & 29.99 & 0.05564 \\
\hline Dibenz[a,j]anthracene & 470 & & 29.99 & 0.063768 \\
\hline
\end{tabular}


Dibenz[a,n]triphenylene,

Naphtho[2,3-g]chrysene

Dibenzo[a,c]naphthacene

Dibenzo[a,e]pyrene

Dibenzo[a,h]pyrene

Dibenzo[a,i]pyrene

Dibenzo[a,j]naphthacene

Dibenzo[a,1]naphthacene

Dibenzo[a,1]pyrene

Dibenzo[b,k]chrysene

Dibenzo[c,g]phenanthrene

Dibenzo[c,1]chrysene

Dibenzo[de,mn]naphthacene

Dibenzo[de,qr]naphthacene

Dibenzo[e,1]pyrene,

Dibenzo[fg,op]naphthacene

Dibenzo[g,p]chrysene
463

540

514

583

553

618

526

437

673

449

482

533

533

624

490
35.95

0.077646

35.95

0.066636

31

30.75

0.059825

30.75

0.052744

30.75

0.055606

35.95

0.058172

35.95

0.068346

25

30.75

0.070366

35.95

0.053418

29.99

0.066867

35.95

0.074663

30.75

0.057692

50

30.75

0.057746

30.75

0.049279

35.95

0.073367 


\begin{tabular}{|c|c|c|c|c|}
\hline Diphenyl & 342 & 18.6 & 17.31 & 0.050688 \\
\hline m-Terphenyl & 360 & 22.6 & 30.69 & 0.08525 \\
\hline m-Xylene & 225 & 11.55 & 9.67 & 0.04294 \\
\hline Naphthacene & 626 & & 24.03 & 0.038387 \\
\hline Naphthalene & 354 & 19 & 12.11 & 0.034248 \\
\hline Naphtho[2,1,8-qra]naphthacene & 546 & & 30.75 & 0.05637 \\
\hline o-Terphenyl & 329 & 17 & 30.69 & 0.093169 \\
\hline o-Xylene & 248 & 13.61 & 9.67 & 0.039023 \\
\hline Pentacene & 563 & 31 & 29.99 & 0.053268 \\
\hline Pentamethylbenzene & 328 & 12.7 & 14.95 & 0.045551 \\
\hline Pentaphene & 530 & & 29.99 & 0.056585 \\
\hline Perylene & 558 & 32.6 & 24.79 & 0.044427 \\
\hline Phenanthrene & 373 & 17.5 & 18.07 & 0.048471 \\
\hline Picene & 641 & 35.19 & 29.99 & 0.046786 \\
\hline p-Quaterphenyl & 587 & 38 & 39.63 & 0.067513 \\
\hline p-Xylene & 286 & 16.8 & 9.67 & 0.033788 \\
\hline p-Terphenyl & 483 & 37 & 30.69 & 0.063527 \\
\hline
\end{tabular}


Pyrene

Triphenylene

Toluene

Butane

Butyl-cyclohexane

Cyclohexane

Decane

Decyl-cyclohexane

Dodecane

Dodecyl-cyclohexane

Ethyl-cyclohexane

Heptane

Heptyl-cyclohexane

Hexadecane

Hexane

Hexyl-cyclohexane

Methyl-cyclohexane
429

472

178

135

198

280

243

271

263

283

162

182

242

291

178

230

147
17.4

18.83

0.043893

25

24.03

0.050911

6.85

7.91

0.044438

4.66

4.27

0.03163

14.1

14.91

0.075303

2.7

13.11

0.046821

28.8

26.83

0.110412

38.6

37.47

0.138266

36.8

34.35

0.130608

45.8

44.99

0.158975

8.3

7.39

0.045617

14.1

15.55

0.08544

22.2

26.19

0.108223

51.5

49.39

0.169725

12.8

11.79

0.066236

22.43

0.097522

6.7

3.63

0.024694 
n-Docosane

n-Eicosane

n-Heneicosane

n-Heptadecane

n-Hetacosane

n-Hexacosane

n-Nonacosane

n-Octacosane

Nonadecane

Nonane

Nonyl-cyclohexane

n-Pentacosane

n-Pentadecane

n-Tetracosane

n-Tetradecane

n-Tetradecyl-cyclohexane

n-Tricosane
315

310

313

290

332

328

334

333

296

220

263

323

283

322

279

298

317
77.3

67.8

63.2

51.2

87.6

91.7

95.8

100.1

50

22

23.07

33.71

83.8

34.6

86.19

45.1

41.87

0.150072

52.51

0.176208

75.7

71.95

0.228413

0.207839

0.217859

0.183276

0.273343

0.265213

0.294222

0.283814

0.204966

0.104864

0.128175

0.257678

0.161237

45.63

0.246801

79.47$$
\text { . }
$$
45.

75.71

0.238833 


$\begin{array}{lcccc}\text { n-Tridecane } & 267 & 28.5 & 38.11 & 0.142734 \\ \text { Octadecane } & 301 & 61.5 & 56.91 & 0.18907 \\ \text { Octane } & 216 & 20.5 & 19.31 & 0.089398 \\ \text { Octyl-cyclohexane } & 253 & & 29.95 & 0.118379 \\ \text { Pentane } & 143 & 8.4 & 8.03 & 0.056154 \\ \text { Pentyl-cyclohexane } & 215 & & 18.67 & 0.086837 \\ \text { Propane } & 85 & 3.52 & 0.51 & 0.006 \\ \text { Propyl-cyclohexane } & 178 & 10.3 & 11.15 & 0.06264 \\ \text { Triacontane } & 337 & 106.32 & 102.03 & 0.30276 \\ \text { Undecane } & 247 & 22.1 & 30.59 & 0.123846\end{array}$

${ }^{\mathrm{a}}$ Experimental melting point values were obtained from Chickos \& Acree et al. ${ }^{31}$ and Chemfinder $^{34}$

${ }^{\mathrm{b}}$ Experimental enthalpy values were obtained from Chickos \& Acree et al. ${ }^{31}$ and Chemfinder $^{34}$

${ }^{\mathrm{c}}$ Calculated enthalpy values were obtained according to the method of Zhao et al. ${ }^{15}$

${ }^{\mathrm{d}}$ Calculated entropy values were obtained using equation 1 , and substituting in the calculated enthalpy values and the experimental melting points. 